\title{
CALCIUM K-LINE PROFILES AS A FUNCTION OF LATITUDE AND SOLAR CYCLE PHASE
}

\author{
JAGDEV SINGH \\ Indian Institute of Astrophysics \\ Rangalore 560034 \\ India
}

ABSTRACT High resolution spectra are being taken in ionized calcium K-line at different latitudes and integrated over the visible 180 degree longitude. These have been analysed to study the variability of the line parameters as a function of latitude. These spectra are being obtained on a regular basis and large data base will be used to study chromospheric rotation, differential rotation in chromosphere, activity and variability of $\mathrm{K}$-line parameters as function of latitude and solar cycle phase. Further, an optical arrangement has been worked out to obtain the spectra along the latitudes of the solar image in the light integrated over longitudes. This involves the unidirectionally focusing of the image in N-S direction.

\section{INTRODUCTION}

Following White and Livingston (1978) the sun as a star has been studied by many observers by monitoring the calcium K-line profiles.

To estimate the 'Active network' component, introduced by Skumanich et al. 1984, a technique has been developed to monitor the calcium K-line profile as a function of latitude and integrated over the visible longitude.

A $36 \mathrm{~m}$ focal length objective forms an image of the sun 34 $\mathrm{cm}$ in diameter at the spectrograph. To obtain the spectrum at a given latitude and integrate over 180 degree longitude, a sun chart, corresponding to the image size and heliographic latitude of the disc centre on that day, is kept near the focal plane of the sun's image in such a way that $\mathrm{N}-\mathrm{S}$ axis marked becomes parallel to the axis of rotation of the image. To integrate the spectra along the longitude, the image is moved 
with a uniform speed from east to west along a given latitude line. It takes 15 seconds to move the image from east to west at the equator during which exposure of the spectrum is made on Kodak 103-aO $35 \mathrm{~mm}$ film. The intensity curves have been normalised at an intensity value of 13 percent at $3935.16 \mathrm{~A}$ on the red wing of calcium K-line given by White and Suemoto (1968).

RESULTS

We have analysed the data of few days obtained between the period Lecember 1985-January 1986. The visible solar surface was almost devoid of plages during that period. The ionized calcium K-line profiles for 80 degree $N$ latitude and solar equator show that the residual intensity in $\mathrm{K}_{3}$ at the poles is about 8 percent as compared to 5 percent at solar equator. The $\mathrm{K}_{2}$ width $0.60 \mathrm{~A}$ at poles in comparison with 0.35 at the solar equator. The analysis of whole data will yield the valuable information about changes in polar regions with solar cycle phase, value of 'Active network' component, differential rotation, Singh and Livingston (1987) and variation of chromospheric rotation with time, Singh and Prabhu (1985).

\section{REFERENCES}

Singh,J. and Prabhu,T.P. 1985, Solar Phys. 97, 203.

Singh,J. and Livingston,W.C. 1987, Solar Phys. 109, 387.

Skumanich,A., Lean,J.L. White,O.R. and Livingston,W.C. 1984, Ap.J. 282, 776.

White,O.R. and Livingston,W.C. 1978, Ap.J. 226, 679.

White,O.R. and Suemoto,Z. 1968, Solar Phys. 3, 523. 